\title{
NASA panel draws up 'road map' to hunt Earth-like planets in space
}

Washington. An ambitious plan to search for Earth-like planets around other stars and perhaps detect life on these, if it exists - is gaining momentum within the US National Aeronautics and Space Administration (NASA), and could become a central part of NASA's science programme for the next decade.

The proposed search strategy, which would begin with ground-based observations and lead early in the next century to large, space-based interferometry arrays, has still to be presented to Daniel Goldin, the administrator of NASA. But Goldin is said to be enthusiastic about the idea in principle, and is expected to unveil details of the plan at an American Astronomical Society meeting in San Antonio, Texas, next month.

A team headed by Charles Elachi of NASA's Jet Propulsion Laboratory (JPL) has spent much of this year drawing up a 'road map' for the search programme, known as Exploration of Neighboring Planetary Systems (ExNPS). Three independent teams, led by Roger Angel of the University of Arizona, Robert Reasenberg of the Smithsonian Astrophysical Observatory and Charles Beichman of JPL, were asked to come up with their own plans for a search.

These sets of proposals were then merged into a single strategy by Elachi's team. This strategy was in turn reviewed by an outside panel headed by Charles Townes of the University of California at Berkeley, who will present his group's assessment of the Elachi report - expected to be favourable - to Goldin next week.

The ExNPS study outlines a phased approach to a planetary search, beginning with the indirect detection of large planets between the sizes of Uranus and Jupiter using ground-based astronometry and radial velocity measurements. Large telescopes with advanced adaptive optics may be able to capture images of Jupiter-sized objects directly, according to the study.

Space-based astrometric interferometers, as well as the use of gravitational "microlenses" to magnify distant star systems, would be able to detect even smaller planets, down to the size of Earth. The final phase of the programme would place a large infrared interferometer in deep space (perhaps as far as Jupiter's orbit) to search for the spectral signature of water, ozone, and other signs of life around Earth-sized planets. The ExNPS search would concentrate on the nearest 1,000 stars, out to a distance of approximately 42 light years.

None of these steps, according to the ExNPS team, requires great leaps in technology, although they do pose engineering challenges. A key guideline given to the Elachi team by NASA headquarters was that the programme be "visionary, but fiscally realistic", according to a draft of the team's report.

How NASA might structure and pay for such a programme is still to be determined. But the agency is already reorganizing its science office, with 'origins' as one of the key themes. Edward Weiler, at present programme scientist for the Hubble Space Telescope, will head the new directorate, which will address a wide range of questions, from galactic evolution to the origins of life.

According to Weiler, ExNPS is only one proposed direction for the office. Another NASA task force has called for the development of an astrometric interferometer in Earth orbit. And a study led by Alan Dressler of the Carnegie Institution for the Association of Universities for Research in Astronomy (AURA) will soon recommend that the agency pursues a next-generation space telescope at least four metres in diameter, which could reveal information on the first billion years of galactic formation. NASA's Goddard Space Flight Center in Maryland has already begun a year-long study to see if such a large space telescope could be built for as little as $\$ 500$ million.

Weiler sees these programmes as complementary, with the astrometric interferometer, for example, fitting snugly into the ExNPS roadmap. But a large space telescope, even a relatively cheap one, could compete with a planetary search for a place in the funding queue.

Weiler emphasizes that "we would like to do this programme without having to look for new money" and that ExNPS "won't be an issue for a Congressional new start for at least one or two years". The earliest the astrometric interferometer could start development, he says, would be 2000 .

Meanwhile, the ground-based search could begin at the Keck Observatory in Hawaii (funded partly by NASA) and at other large observatories now in the planning stages. The Space Infrared Telescope Facility (SIRTF), scheduled for launch in 2002, also contributes to the ExNPS programme, says Weiler, as it tests cryogenic technologies needed for a space-based interferometer and provides critical data on the dust surrounding candidate stars.

Weiler says that it is too early to estimate the overall cost of ExNPS. But he believes it could be carried out for between 10 and 20 per cent of NASA's space science budget over the next 20 years. That number could be lower still if international partners are brought in, according to members of the ExNPS team. The European Space Agency has a similar concept for a space infrared interferometer, called Darwin, under study.

But aside from funding concerns, the ExNPS report is expected to come to be seen as a milestone in NASA's history, namely as the space agency's official statement that the detection of Earth-like planets is now both scientifically and technically feasible.

Tony Reichhardt 